\title{
Management of the Sequelae of Severe Congenital Abdominal Wall Defects
}

\author{
Sara Fuentes ${ }^{1}$, Eunate Marti ${ }^{2}$, Maria-Dolores Delgado ${ }^{2}$, Andres Gomez ${ }^{2}$ \\ ${ }^{1}$ General Pediatric Surgery Department, Nino Jesus University Hospital, Madrid; ${ }^{2}$ Pediatric Plastic Surgery Department, 12 de Octubre \\ University Hospital, Madrid, Spain
}

Background The survival rate of newborns with severe congenital abdominal wall defects has increased. After successfully addressing life-threatening complications, it is necessary to focus on the cosmetic and functional outcomes of the abdominal wall.

Methods We performed a chart review of five cases treated in our institution.

Results Five patients, ranging from seven to 18 years of age, underwent the following surgical approaches: simple approximation of the rectus abdominis fascia, the rectus abdominis sheath turnover flap, the placement of submuscular tissue expanders, mesh repair, or a combination of these techniques depending on the characteristics of each individual case.

Conclusions Patients with severe congenital abdominal wall defects require individualized surgical treatment to address both the aesthetic and functional issues related to the sequelae of their defects.

Keywords Gastroschisis / Umbilical hernia / Abdominal wall / Abdominal muscles / Hernia, ventral
Correspondence: Sara Fuentes Nino Jesus University Hospital, Avda Menéndez Pelayo 65, 28009 Madrid, Spain

Tel: +34-64-977-0758

Fax: +34-91-503-5900

E-mail: sarafuentesc@yahoo.es

This article was presented as a poster at the XLVIII Meeting of the Spanish Society of Pediatric Surgery on May 2009, in Seville, Spain.

No potential conflict of interest relevant to this article was reported.

Received: 20 Jan 2016 • Revised: 18 Feb 2016 • Accepted: 29 Mar 2016

pISSN: 2234-6163 • elSSN: 2234-6171 • http://dx.doi.org/10.5999/aps.2016.43.3.258 • Arch Plast Surg 2016;43:258-264

\section{INTRODUCTION}

Gastroschisis (GS) and omphalocele (OC) are the most common congenital abdominal wall defects (CAWDs). Their management involves a multidisciplinary team for both the initial treatment and for long-term follow-up. These defects occur with a wide range of severity, and the more severe examples of these defects present a medical and surgical challenge. Giant OC is defined as a defect involving the liver that measures at least $4.5-5 \mathrm{~cm}$. The abdominal defect present in GS is usually smaller, which may result in damage of the herniated content. Both are associated with a severe disproportion between the abdominal cavity and the viscera, as well as with respiratory problems [1].
Table 1 summarizes the main characteristics of these entities along with their management and outcomes.

Up to $37 \%$ of patients with severe CAWDs, including GS or OC, report concerns or self-esteem problems regarding their scar $[2,3]$. In most cases, the abdominal wall contains defects involving muscular layers or the fascial component, which may have a negative impact on physical exercise or on pregnancy in females. The aim of this report was to present five cases treated in our department.

\section{METHODS}

We performed a review of cases involving the sequelae of severe CAWDs treated in our institution. We obtained information re- 
Table 1. Summary of the main features of gastroschisis and omphalocele

\begin{tabular}{|lll|}
\hline Features & Omphalocele & Gastroschisis \\
\hline Site of the defect & Central umbilical & Right paraumbilical \\
Sac & Always present & Never present \\
Anomalies of rotation and fixation & Present & Present \\
Gender & More frequent in females & Equal \\
Associated anomalies & Frequent (heart anomalies) & Rare \\
Intestinal atresia & Rare & Up to 10\% \\
\hline
\end{tabular}

\section{Fig. 1. Approximation of the rectus abdominis}

This figure shows how the technique is performed easily when the distance is minimal.

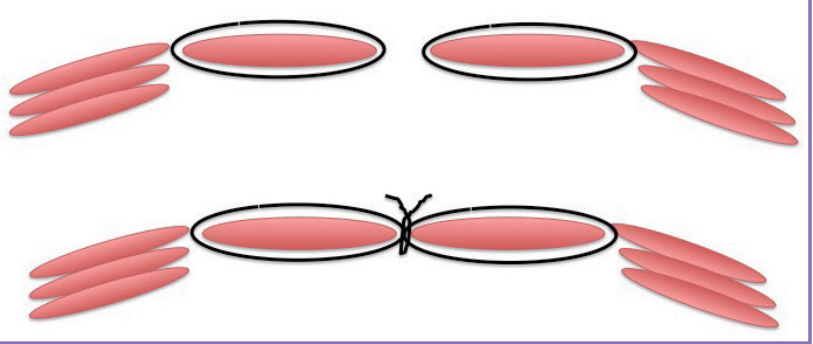

garding the original defect, initial management, follow-up, and surgical approach in our department, as well as the final outcome.

When abdominal wall reconstruction was planned, the main emphasis was on the functional anatomy of the abdominal wall. The size and extent of the defects were paramount in choosing the surgical approach, and for the widest defects, different techniques were considered, with the final decision made during surgery. The most severe cases were studied by magnetic resonance (MR) imaging in order to assess the exact position and development of the muscular layers.

Our first-choice approach, whenever possible, was approximation of the rectus abdominis muscle (Fig. 1). Care must be taken during dissection due to the strong adhesions usually found under the defect and the underlying bowel. It is also important to properly identify the edges of the defect and to extend the suture beyond the cranial and caudal margins, in order to avoid weaknesses at these points. Once dissection is performed, it is important to assess the feasibility of direct closure, ensuring that the abdomen does not experience high pressure, which could result in early and late complications.

For cases where simple approximation is not possible, different approaches have been proposed. In our institution, the rectus abdominis muscle sheath turnover flap is the technique of choice $[4,5]$. However, all patients selected for this intervention are routinely informed and measures are taken for the possibility of placing tissue expanders if closure is not feasible during the

\section{Fig. 2. Rectus abdominis muscle sheath turnover flap}

The black lines represent the fascia that is divided and turned to meet medially (arrow, incision in the fascia).

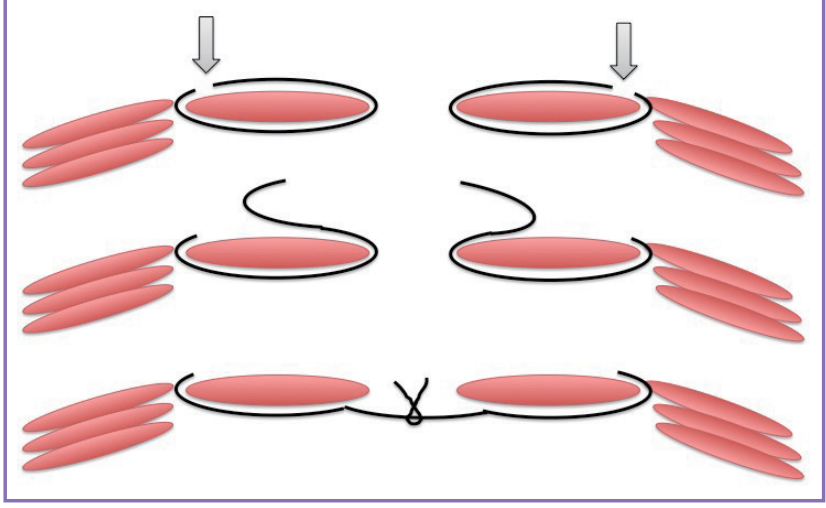

Fig. 3. Submuscular tissue expanders

The figure demonstrates how tissue expanders enlarge the available tissue in order to allow closure (arrow, approximation of edges).
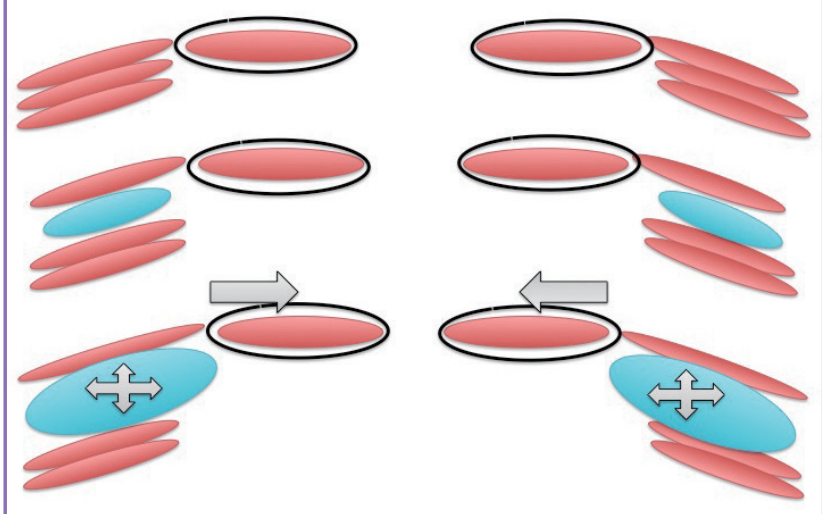

surgery. The first part of the intervention is probably the more delicate part, as described above, due to the severe adhesions that can be found and the superficial location of the bowel under the skin. Once the rectus abdominis muscles are identified, traction is placed and the defect is measured to ensure that tissue expanders are not needed. Subsequently, a flap is developed, opening the anterior fascia close to the lateral border of the sheath. Both layers are closed in the midline and the rest of the abdomen is closed (Fig. 2).

When abdominal closure is not likely to be accomplished using this technique, crescent-shaped submuscular tissue expanders are placed and progressively filled for a period of approximately six months before the definitive repair is performed (Fig. 3) [6].

In the second operation, the expanders are retrieved and a rectus abdominis muscle sheath turnover flap is usually needed to finally close the abdominal wall.

We considered the use of prosthetic mesh in more complex cases, but do not recommend its use. However, it must be taken 
into consideration in the few cases in which closure would not be possible even with the combination of the above-described techniques (Fig. 4) [7].

\section{RESULTS}

The cases are summarized in Table 2.

\section{Approximation of the rectus abdominis muscle}

Two girls with OC, seven and eight years of age, presented with similar central defects. The first defect measured $5 \times 3 \mathrm{~cm}$ and the second $6.5 \times 3 \mathrm{~cm}$. Both lacked an umbilicus.

They were treated successfully by approximation of the rectus abdominis muscles to the xiphoid-pubic line using an interrupted monofilament non-absorbable 3-0 suture. Dense adhesions were found under that area. Resection of scar tissue and neoumbilicoplasty were also performed.

\section{Fig. 4. Turnover flap with prosthetic mesh}

The figure indicates how mesh is used when a tension-free closure is not possible (arrow, incision in the fascia).

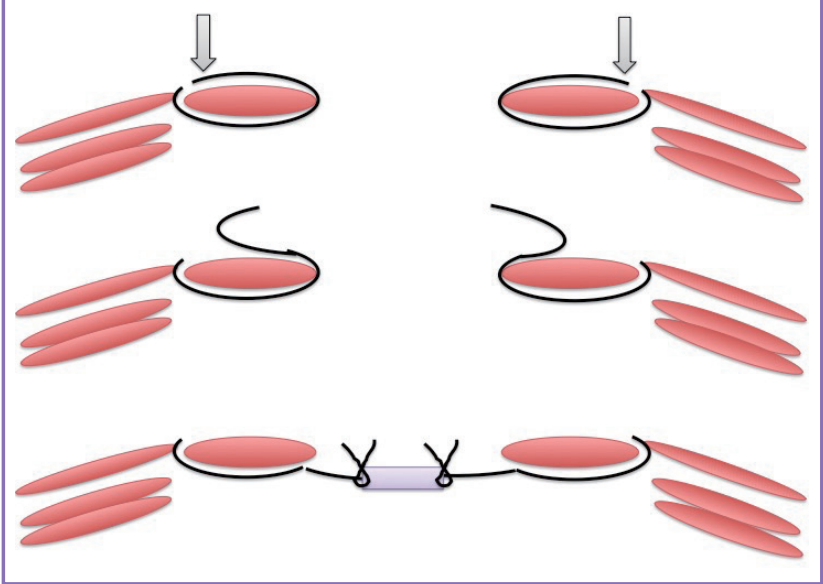

\section{Rectus abdominis muscle sheath turnover flap}

A 14-year-old male patient with GS presented with an abdominal wall defect measuring $15 \times 8 \mathrm{~cm}$ with scarred skin and absence of the umbilicus. The MRI scan showed that the rectus abdominis muscles were present.

During the surgery, dense adhesions were found under the scar and it was not possible to approximate both rectus abdominis muscles. Therefore, in this case we performed a rectus abdominis muscle sheath turnover flap with a running non-absorbable 3-0 suture from the xiphoid to the pubis, resection of the scarred skin, and neoumbilicoplasty (Fig. 5).

\section{Crescent-shaped submuscular tissue expanders}

The last two cases were two girls with giant $\mathrm{OC}$ who underwent staged reduction in the neonatal period and presented with severe abdominal wall defects with scarred skin covering almost the entire abdomen. Both of them presented with slightly hypoplastic rectus abdominis muscles, displaced laterally.

The first girl was 11 years old when we started reconstruction. She underwent the placement of crescent-shaped tissue expanders between the internal oblique and transversus abdominis muscle. Two months later, the expanders were removed and the abdominal wall was repaired using a rectus abdominis muscle sheath turnover flap from the xiphoid to the pubis. It was necessary to add a Goretex mesh of $4 \times 3 \mathrm{~cm}$ on the cranial third of the defect due to the lack of tissue for primary closure. The skin was remodeled and neoumbilicoplasty was performed. She presented with extrusion of the mesh two months later. The cosmetic result is currently acceptable, and we are considering the possibility of re-implanting a new mesh for the remaining proximal defect (Fig. 6).

The second girl underwent reconstruction when she was 18 years old. She also underwent the placement of crescent-shaped tissue expanders between the internal oblique and transversus abdominis muscle.

Table 2. Cases included in this study

\begin{tabular}{|c|c|c|c|c|c|c|c|c|}
\hline Patient & CAWD & $\begin{array}{c}\text { Primary } \\
\text { management }\end{array}$ & Follow-up & $\begin{array}{l}\text { Defect } \\
\text { size }(\mathrm{cm})\end{array}$ & $\begin{array}{l}\text { Age } \\
\text { (yr) }\end{array}$ & Type of repair & Complications & Final result \\
\hline 1 & OC & Primary closure & Ventral hernia & $5 \times 3$ & 7 & Approximation of rectus abdominis & No & Satisfactory \\
\hline 2 & $\mathrm{OC}$ & Primary closure & Ventral hernia & $6.5 \times 3$ & 8 & Approximation of rectus abdominis & No & Satisfactory \\
\hline 3 & GS & $\begin{array}{r}\text { Stage reduction and } \\
\text { secondary closure }\end{array}$ & Ventral hernia & $15 \times 8$ & 14 & Rectus abdominis sheath turnover flap & No & Satisfactory \\
\hline 4 & $\mathrm{OC}$ & $\begin{array}{r}\text { Stage reduction and } \\
\text { secondary closure }\end{array}$ & Ventral hernia & $23 \times 19$ & 11 & $\begin{array}{l}\text { Submuscular tissue expanders+rectus } \\
\text { abdominis sheath turnover flap+ } \\
\text { goretex mesh }\end{array}$ & $\begin{array}{l}\text { Extrusion of the } \\
\text { mesh }\end{array}$ & Ventral hernia \\
\hline 5 & OC & $\begin{array}{r}\text { Stage reduction and } \\
\text { secondary closure }\end{array}$ & Ventral hernia & $20 \times 11$ & 18 & $\begin{array}{l}\text { Submuscular tissue expanders+direct } \\
\text { closure }\end{array}$ & $\begin{array}{l}\text { Diastasis of the } \\
\text { rectus abdominis }\end{array}$ & $\begin{array}{l}\text { Rectus abdominis } \\
\text { sheath turnover flap }\end{array}$ \\
\hline
\end{tabular}


Fig. 5. A case of rectus abdominis muscle sheath turnover flap

(A) A child with a giant ventral hernia following gastroschisis closure. (B) The fascia is already opened (hemostats on the right side). The superior hemostat holds the left edge of the peritoneum that has to be closed before approximating the fascia. (C) The intradermic suture and neoumbilicoplasty can be appreciated.
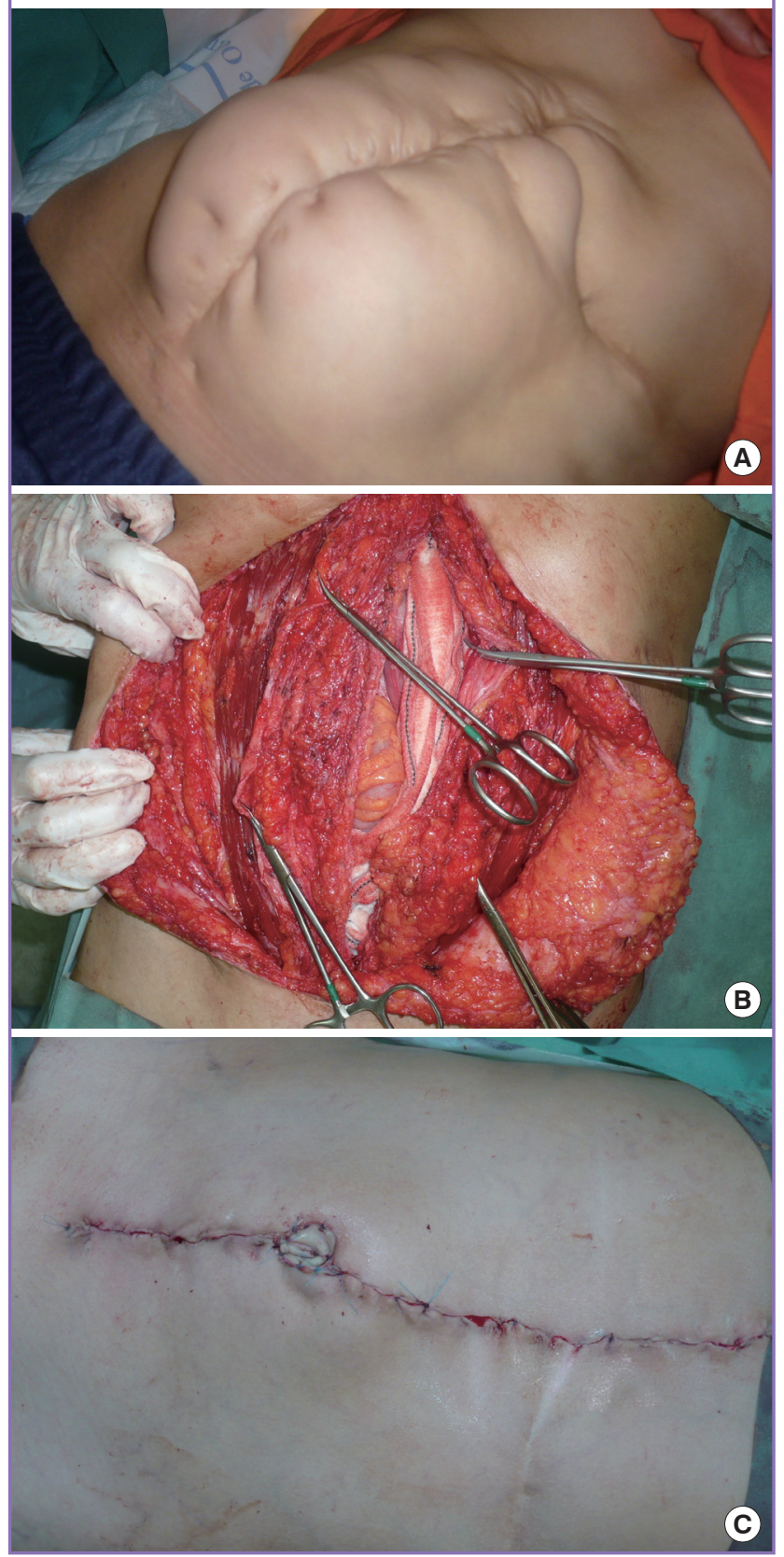

Two months later she underwent removal of the expanders and direct closure by approximating the rectus abdominis muscles to the xiphoid-pubic line as described above, removal of the scarred skin, and neoumbilicoplasty. Eighteen months later, she presented with diastasis of the rectus abdominis muscles that interfered with her professional athletic career, and we therefore performed a rectus abdominis muscle sheath turnover flap (Fig. 7).
Fig. 6. A case of submuscular tissue expanders

(A) This patient underwent staged closure, and abdominal complications occurred, including enterocutaneous fistulae, during the neonatal period. (B) Crescent-shaped expanders are visible and the scar tissue to be removed is marked. (C) Some scar tissue is still present, but it has been minimized and the functional outcome was optimal.
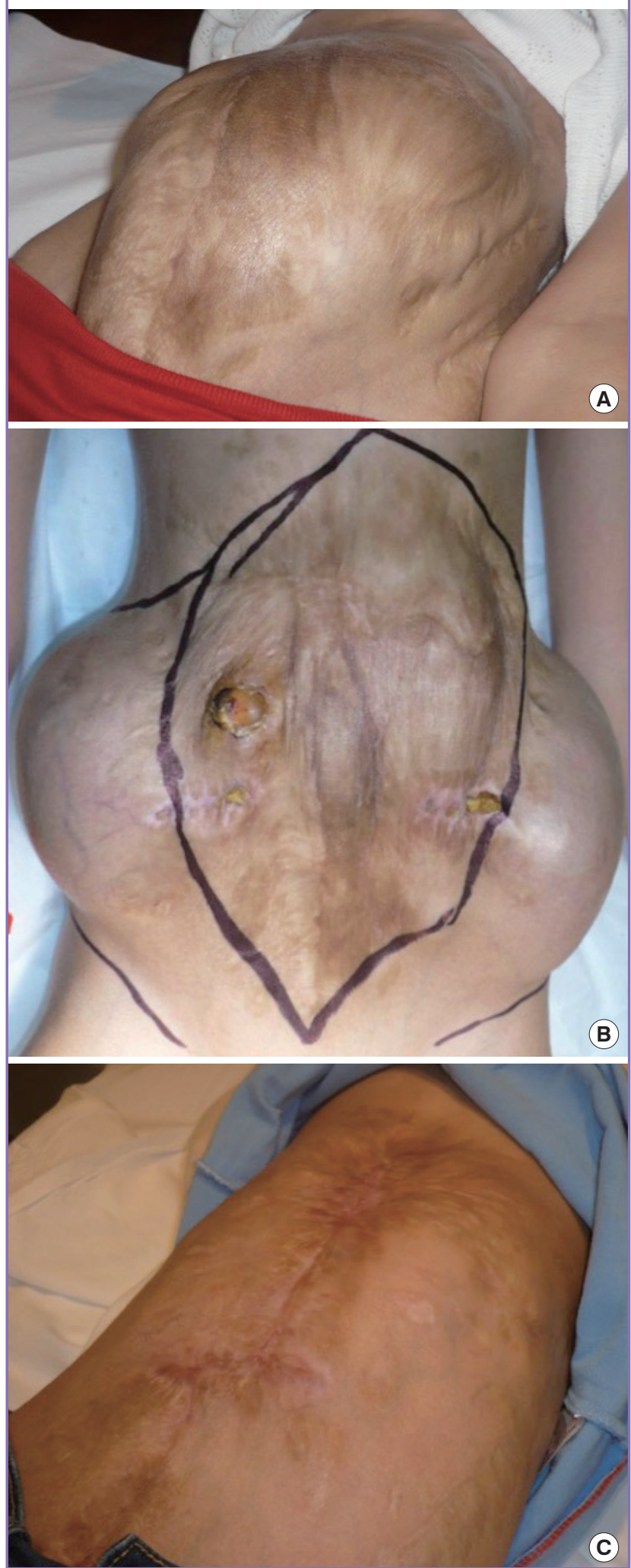
Fig. 7. A case of diastasis of the rectus abdominis muscles after tissue expanders

(A) This patient underwent staged closure, and the image shows the ventral hernia and the separation of the rectus abdominis muscles. (B) Scarring is minimal and the functional outcome was optimal.

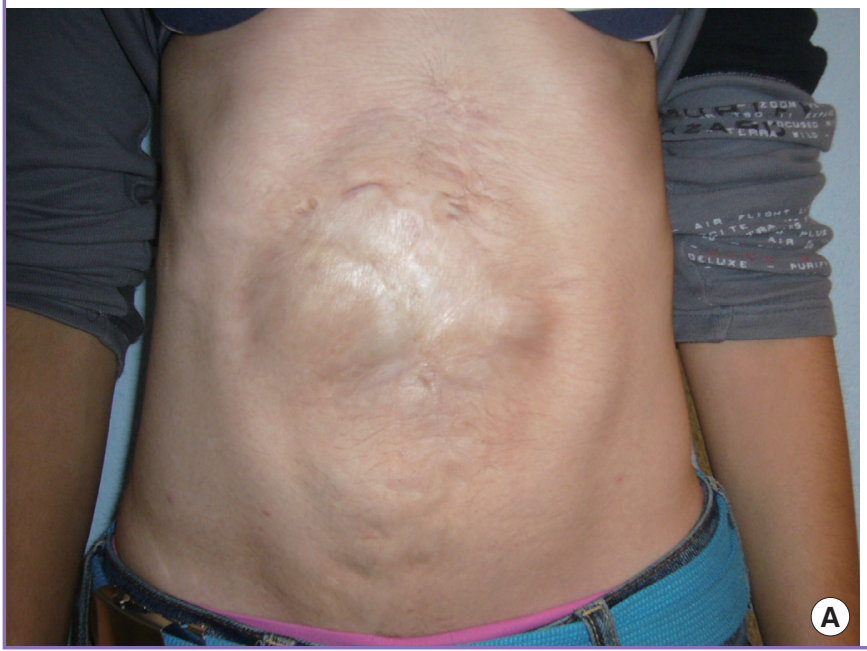

\section{DISCUSSION}

CAWDs have an overall incidence of $1 / 3,000$ births for $\mathrm{OC}$ and $1 / 5,000$ births for GS [1]. Cases of GS have increased, whereas the incidence of $\mathrm{OC}$ seems to have remained stable. Both conditions have different embryological and pathophysiological characteristics, but they share long-term outcomes regarding the functional and anatomical development of the abdominal wall.

OC most commonly occurs due to an alteration of abdominal wall formation in which the abdominal viscera fail to return to the peritoneal cavity after the eleventh week of gestation. The defect, therefore, is covered by a sac. The abdominal muscles are usually hypoplastic and displaced laterally. The intestine itself is normal, but the condition is associated with a high rate of associated anomalies, such as cardiac defects. Giant OC is defined as a defect of $4 \mathrm{~cm}$ or more than includes the liver or the spleen along with hollow viscera.

GS, in contrast, is a defect of the entire abdominal wall. It is usually located to the right of the umbilical cord, and, although its etiology is unclear, it is thought to be related to a vascular event during the early stages of embryological development. The herniated loops are not covered by a sac and the abdominal wall muscles are affected on the right side. Associated systemic anomalies are not as frequent as they are in OC, but intestinal malformations are common, such as intestinal atresia or secondary dam-

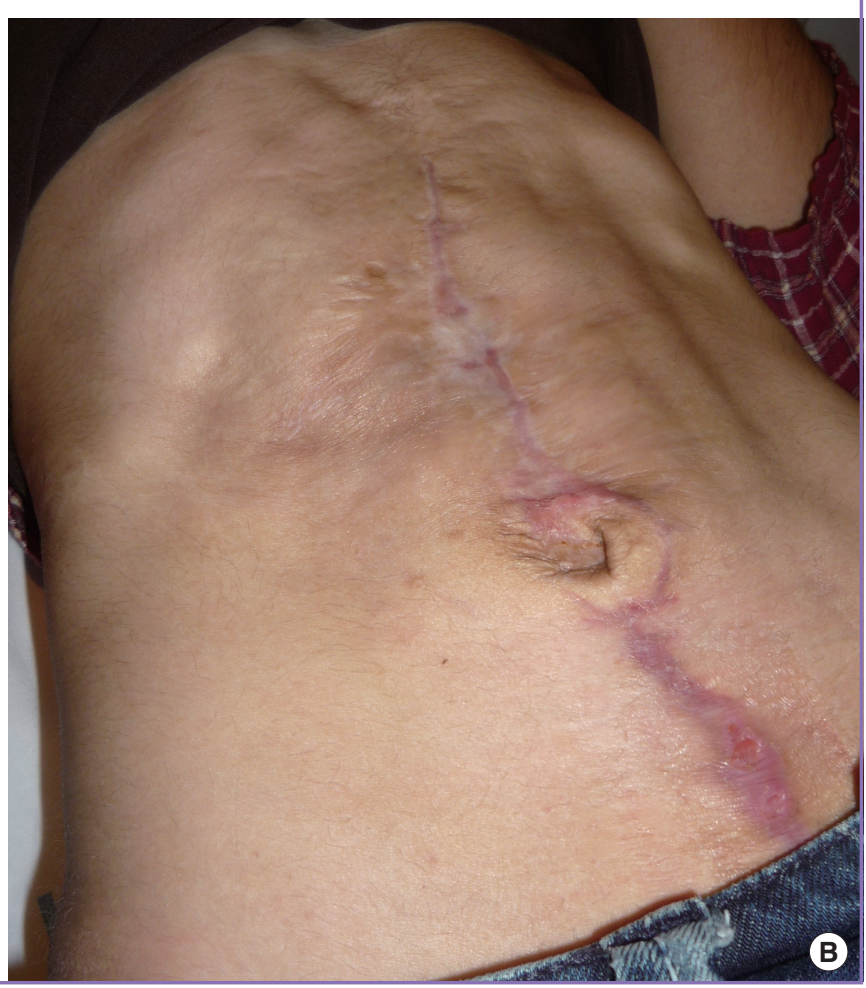

age due to exposure of the amniotic fluid or compression by the small size of the abdominal wall defect [1].

The first step in the management of both conditions is prenatal and perinatal care. After delivery, the main concerns are stabilizing the patient and ruling out associated malformations. Subsequently, surgical procedures aim to reduce and close the defect. The way this can be accomplished depends mainly on the amount of herniated viscera and the distensibility of the abdominal wall. When possible, reduction of the structures and primary closure is performed. If the abdominal pressure increases over $15 \mathrm{~mm} \mathrm{Hg}$, the risk of ventilatory and hemodynamic complications, along with the possibility of developing abdominal compartment syndrome, makes this approach less favored. In such cases, an extracorporeal bag or silo is sutured around the defect and the abdominal contents are gently reduced into the peritoneal cavity over a period of seven to 10 days. After that, the silo is removed and the abdominal wall is repaired. For giant $\mathrm{OC}$, a delayed approach has also been described, involving the treatment of the sac with topical agents in order to make it epithelize, repairing the defect later in a second-stage operation $[8,9]$.

Patients with severe CAWDs present with a wide variety of cosmetic and functional sequelae on their abdominal walls, which may have consequences for their self-esteem or for their everyday lives, especially when playing sports or considering pregnancy $[10]$. 
CAWDs are more common in adult patients than in children as a consequence of postoperative or traumatic damage. Even for this group of patients, however, experience is limited and scarce evidence exists regarding the optimal surgical management of such cases $[7,11,12]$. In the pediatric population, the use of meshes for repair should be avoided, as they lead to complications as the child grows up. Nevertheless, meshes sometimes are the only possible way to close a defect, alone or in combination with other techniques.

When evaluating a child with sequelae of severe CAWDs, a physical examination is essential for deciding which technique will be most suitable. Ultrasound and MR imaging may play a role in studying the characteristics of the abdominal wall. However, the abdominal wall muscles are present in the vast majority of cases, although they are hypoplastic and displaced. The age at which the procedure is performed plays an important role as well. We prefer to treat these patients early, once their main medical problems have been resolved and before the displacement of the muscles increases with normal growth. The extent of the defect provides information about which technique is likely to be more useful. In our experience, defects with a transverse length of less than $5 \mathrm{~cm}$ can be successfully managed by a simple approximation of the rectus. Between $5 \mathrm{~cm}$ and $8 \mathrm{~cm}$, a rectus abdominis turnover flap would be our first choice. However, for the widest defects, we always consider the possibility of submuscular tissue expanders, and both the family and the patients are informed that during surgery, if we feel that it will not be safe to perform the turnover flap, we will place tissue expanders. The abdominal wall component separation technique has been reported to be safe and effective for the management of CAWDs, including giant OC [13]. We have not performed this procedure, but the results reported by other authors seem promising [14].

The use of a prosthetic mesh is widely accepted in adult patients $[7,11]$. However, concerns have been raised with regard to the pediatric population about the effect of growth on the final outcomes of the mesh in terms of displacement or extrusion. Whenever it is possible, we prefer not to use a prosthetic mesh, although new biological meshes are being developed and could be of use in the pediatric population [15].

It is essential that these patients undergo close long-term follow up in order to promptly identify and resolve any complications that might arise during growth, whether functional or cosmetic.

Patients with severe CAWDs present with a wide variety of cosmetic and functional sequelae that may have a significant impact on their self-esteem or their future lives.

Each of these patients must be considered and studied individually in order to choose the best technique or combination of techniques capable of providing them with the optimal cosmetic and functional outcomes.

\section{REFERENCES}

1. Gamba P, Midrio P. Abdominal wall defects: prenatal diagnosis, newborn management, and long-term outcomes. Semin Pediatr Surg 2014;23:283-90.

2. Koivusalo A, Lindahl H, Rintala RJ. Morbidity and quality of life in adult patients with a congenital abdominal wall defect: a questionnaire survey. J Pediatr Surg 2002;37:1594601.

3. Henrich K, Huemmer HP, Reingruber B, et al. Gastroschisis and omphalocele: treatments and long-term outcomes. Pediatr Surg Int 2008;24:167-73.

4. Kushimoto S, Yamamoto Y, Aiboshi J, et al. Usefulness of the bilateral anterior rectus abdominis sheath turnover flap method for early fascial closure in patients requiring open abdominal management. World J Surg 2007;31:2-8.

5. DeFranzo AJ, Kingman GJ, Sterchi JM, et al. Rectus turnover flaps for the reconstruction of large midline abdominal wall defects. Ann Plast Surg 1996;37:18-23.

6. Paletta CE, Huang DB, Dehghan K, et al. The use of tissue expanders in staged abdominal wall reconstruction. Ann Plast Surg 1999;42:259-65.

7. Butler CE, Baumann DP, Janis JE, et al. Abdominal wall reconstruction. Curr Probl Surg 2013;50:557-86.

8. Akinkuotu AC, Sheikh F, Olutoye OO, et al. Giant omphaloceles: surgical management and perinatal outcomes. J Surg Res 2015;198:388-92.

9. Pandey V, Gangopadhyay AN, Gupta DK, et al. Non-operative management of giant omphalocele with topical povidoneiodine and powdered antibiotic combination: early experience from a tertiary centre. Pediatr Surg Int 2014;30:407-11.

10. Hwang PJ, Kousseff BG. Omphalocele and gastroschisis: an 18-year review study. Genet Med 2004;6:232-6.

11. Leppaniemi A, Tukiainen E. Planned hernia repair and late abdominal wall reconstruction. World J Surg 2012;36:511-5.

12. Eriksson A, Rosenberg J, Bisgaard T. Surgical treatment for giant incisional hernia: a qualitative systematic review. Hernia 2014;18:31-8.

13. van Eijck FC, de Blaauw I, Bleichrodt RP, et al. Closure of giant omphaloceles by the abdominal wall component separation technique in infants. J Pediatr Surg 2008;43:246-50.

14. van Eijck FC, van Vlimmeren LA, Wijnen RM, et al. Functional, motor developmental, and long-term outcome after the component separation technique in children with giant omphalocele: a case control study. J Pediatr Surg 2013;48: 
525-32.

15. Meyer T, Schwarz K, Ulrichs K, et al. A new biocompatible material (Lyoplant) for the therapy of congenital abdominal wall defects: first experimental results in rats. Pediatr Surg Int 2006;22:369-74. 